\title{
Dynamics of Patterns on Elastic Hypersurfaces. Part II. Wave Mechanics of Flexural Quasi-Particles
}

\author{
C. I. Christov \\ Dept. of Mathematics, University of Louisiana at Lafayette, Lafayette, LA 70504-1010
}

\begin{abstract}
In the first part of this work, the shear wave phenomena in an elastic 3D continuum are investigated and an analogy to Maxwellian electrodynamics is shown. In the present part, the model is extended assuming the continuum to be a momentum-supporting hypersurface in $4 \mathrm{D}$ space (a hypershell). The transverse (flexural) deformations of the shell are governed by a Generalized Nonlinear Dispersive Wave Equation (GDWE) of Schrödinger type. The Hamiltonian structure of the model is discussed. The solitary wave solutions are shown to fit the concept of quasi-particles. The concept of pseudo-mass is introduced and the Newtonian mechanics for the centers of quasiparticles/solitons is derived. Numerical examples of the shapes in 2D are presented. The presence of attractive force between the localized elevations of the surface is discussed and shown to depend on the distance between them as $|\vec{x}|^{-2}$.
\end{abstract}

Keywords: Unified Field Theory, Wave Mechanics

PACS: 03.50.-z, 46.70.De

\section{A NON-PROBABILISTIC APPROACH TO WAVE MECHANICS}

De Broglie proposed to explain certain diffraction and interference patterns of electron beams by attaching a wave to a particle. Schrödinger, guided by some heuristic considerations of the principles of wave propagation, derived an equation for the wave function. Schrödinger's equation of wave mechanics is now widely accepted for describing properties of particles (see, for the historical review, [1]). The important characteristic of the Schrödinger equation is that it is not a part of Maxwell's equations. Hence if we are to look for an analogy between the known physical phenomena and the elastic continuum we must transcend the three dimensions because the presence of an additional variable is connected with the existence of an additional dimension. It was first suggested by Hinton [2] that the fourth dimension is not tangible because the 4D thickness of the material world is so minute that it cannot be appreciated.

The derivation of Schrödinger's equation for the purposes of the wave mechanics did not involve physical stipulations of the particular mechanical properties of the field, but Schrödinger [3] did point out in his original paper that the equation at which he had arrived at, was the Euler-Bernoulli equation for the deformation of elastic plates. Since then, the Schrödinger equation proved successful in modeling different atomic phenomena. Now, it is considered as an experimentally established fact that the "master" equation of the wave mechanics is from the genus of the generalized dispersive wave equations (GDWE). In this section we try to come up with a mechanical construct which gives us a fourth order nonlinear dispersive equation, so we can provide arguments for 
the physical basis for the Schrödinger's equation.

Following this line of reasoning, we propose to extend the analogy introduced in Part I. We couple the equations for the laminar components with an equation for the flexural deformation along the fourth dimension. When an observer is situated entirely in the 3D middle surface, the presence of the fourth dimension will be appreciated as some additional forces due to the flexural deformations.

In this paper we assume that the dispersive "master equation" of the wave mechanics is not about an "associated" probability wave, but about a real material wave on the surface of a thin 3D hypersurface (a hyperplate or hypershell).

\section{Schrödinger Equation Revisited}

To delineate the analogy to plate theory we begin with the linear Schrödinger equation for a complex wave function $\psi$ which reads

$$
i \frac{\partial \psi}{\partial t}+\hbar \Delta \psi-\chi \psi=0, \quad i \equiv \sqrt{-1}, \quad \psi \equiv \psi_{1}+i \psi_{2},
$$

where $\hbar$ is the Plank constant and $\chi$ is allegedly connected to the potential of acting forces. In terms of the real (or the imaginary) part of wave function

$$
\frac{\partial^{2} \psi_{1}}{\partial t^{2}}+\hbar^{2} \Delta \Delta \psi_{1}-2 \chi \hbar \Delta \psi_{1}+\chi^{2} \psi_{1}=0
$$

For $\chi=0$ this is nothing else but the Euler-Bernoulli equation for flexural deformation of an elastic plate with a stiffness proportional to $\hbar^{2}$. Depending on the sign of $\chi$, different interpretations of Eq. (2) are possible.

\section{Governing Equations for 3D Shells in the 4D Geometrical Space}

Consider a very thin elastic structure (a shell) of a 4D material. A 3D momentum supporting hypersurface is a mathematical abstraction for this kind of material structure. Assume also that the shear Lamé coefficient is much smaller than the dilational one (the 4D material is virtually incompressible).

In the middle 3-surface of the shell, the equations for the so-called laminar components are the equations used in Part I to show the analogy to the Maxwell electrodynamics. Consider now the component of the displacement, $\zeta$, in the 4-th dimension. Contrary to the shell theory in technological applications, we consider here the limiting case $L \gg h$ when the deflections are small, the strains (gradients) are of unit-order, and curvatures are large. Such an object is geometrically strongly nonlinear. Under the above assumptions a geometrically nonlinear theory of the very thin but very stiff shells can be derived. We follow in this instance $[4,5,6,7]$, namely

$$
\mu_{0} \frac{\partial^{2} \zeta}{\partial t^{2}}=F^{(4)}+D\left[-\Delta \Delta \zeta-(\Delta \zeta)^{3}\right]+\sigma_{0} \Delta \zeta-\frac{a}{R} \zeta
$$


Dimensionless form is obtained by using the scales

$$
\zeta=L \zeta^{\prime}, \quad x=L x^{\prime}, \quad t=L c_{f}^{-1} t^{\prime}, \quad c_{f}=\sqrt{\left|\sigma_{0}\right| \mu_{0}^{-1}}, \quad \beta=D\left|\sigma_{0}\right|^{-1} L^{-2},
$$

where $L$ is the characteristic length-scale of the waves, $\mu_{0}$ is the density of the $4 D$ material, $\sigma_{0}$ is the membrane tension and $F^{(4)}$ is a $4 \mathrm{D}$ body force (e.g., normal pressure from the fourth dimension). Here $c_{f} \neq c$.

$$
\frac{\partial^{2} \zeta^{\prime}}{\partial t^{2}}=\beta\left[-\Delta \Delta \zeta^{\prime}-\left(\Delta \zeta^{\prime}\right)^{3}\right]+\varepsilon \Delta \zeta^{\prime}-\kappa \zeta^{\prime}+F,
$$

where $\varepsilon= \pm 1$ and $\kappa$ is the dimensionless undisturbed curvature of the shell. $\beta$ is the dimensionless stiffness of the shell. We consider such a deformation for which $\beta \sim O(1)$. for definiteness we set $\beta=1$.

The linear part of Eq. (5) has the form Eq. (2). The membrane tension and the mean curvature define the potential $\chi$.

Note that the cubic nonlinearity appear in the fourth-order equation for the real part. In this sense the above equation is not exactly what is called NLSE, but the latter has never been derived for the wave mechanics, but only for nonlinear optics. So one can assume that the newly derived GDWE is in very good qualitative agreement with the spirit of Schrödinger's equation.

In order to assess membrane tension in the above model, we can assume that the 3D shell is spherical and subjected to constant normal pressure from the adjacent 4D space outside the shell. Depending on whether the pressure is positive or negative, the resulting membrane tension can be negative or positive. If $V_{g}$ is the magnitude of the external pressure then, $\sigma_{0}=-V_{g} R^{-1}$, where $R$ is the radius of the shell. When a spherical shell is considered the above model has to be augmented with a liner term with coefficient proportional to the mean curvature $R^{-1}$ (see [8]). A fairly general form of a generic Boussinesq equation describing the model has the dimensionless form

$$
\frac{\partial^{2} w}{\partial t^{2}}=-\kappa w+\Delta\left[\varepsilon w-w^{3}-\Delta w\right]
$$

where $w$ stands for the dimensionless deflection.

Buckling the undisturbed spherical surface (the vacuum state), gives birth to localized creatures - solutions. Wave Mechanics is about nonlinear eigenvalue problems. Hence it is by necessity Quantum.

\section{Least-Action Principle Formulation}

Consider trivial boundary conditions for $w=0, \Delta w=0$, at $x \in \partial D$ which correspond to an isolated system. Then we prove that the governing equation (5) provides the minimum to the action integral $L \stackrel{\text { def }}{=} \int_{D} \mathscr{L} \mathrm{d}^{3} x$, where

$$
\mathscr{L}=-\frac{1}{2}\left[-\left(\nabla w_{t}\right)^{2}+\varepsilon(\Delta w)^{2}+\kappa(\nabla w)^{2}-\frac{1}{2} \beta(\Delta w)^{4}+\beta(\nabla \Delta w)^{2}\right] .
$$


It is readily shown that for asymptotically vanishing wave profiles and for a very large region $D$, the volume of the wave (sometimes called "wave mass"), and the energy are given by

$$
V \equiv \int_{D} \Delta w \mathrm{~d}^{3} x, \quad H=\int_{D}\left[\frac{1}{2}\left(\nabla w_{t}\right)^{2}-\mathscr{L}\right] \mathrm{d}^{3} x,
$$

while for the wave momentum we can derive

$$
\vec{P} \equiv \int_{D} w_{t} \nabla \Delta w \mathrm{~d}^{3} x=-\int_{D}\left(\nabla w_{t}\right) \cdot(\nabla \nabla w) \mathrm{d}^{3} x+\oint_{\partial D} w_{t} \Delta w \mathrm{~d} \vec{s}
$$

where the last integral vanishes due to the asymptotic boundary conditions.

The wave volume and energy are conserved, namely $\dot{V}=0, \dot{H}=0$. For the wave momentum $\vec{P}$ we have

$$
\begin{aligned}
\frac{d \vec{P}}{d t}=\oint_{\partial D} \vec{n}\left[-\frac{1}{2}\left(\nabla w_{t}\right)^{2}-\frac{1}{2}(\Delta w)^{2}+(\nabla w)^{2}-\frac{\beta}{4}(\Delta w)^{4}\right. & \left.+\frac{\beta}{2}(\nabla \Delta w)^{2}\right] \mathrm{d} s \\
& -\oint_{\partial D} \beta(\nabla \Delta w) \frac{\partial \Delta w}{\partial n} \mathrm{~d} \vec{s}
\end{aligned}
$$

where the r.h.s. is called pseudoforce. $P$ is conserved if the pseudoforce is zero. For an isolated system of localized waves, the latter is exactly the case because of the asymptotic conditions at infinity (very large regions $D$ ).

\section{SOLITARY WAVES AS QUASI-PARTICLES}

\section{Boussinesq Paradigm}

A major deficiency of the De-Broglie-Schrödinger wave mechanics is that it tacitly assumes that the wave packets are localized but such a packet is not a solution per se of any kind of linear equation. Even in the cases with radial symmetry when localization is observed for the solutions of linear equations, the concept is still not justified because the dispersion will inevitably destroy such a localized wave packet. It turns out that the analogy between waves and particles can be extended farther only if one admits nonlinearity in the model.

Russell [9] discovered a permanent wave (the "Great Wave") on the surface of a shallow water layer (channel). Boussinesq [10] came up with a fundamental idea: the balance between the nonlinearity and dispersion makes the shape of the wave permanent. The equation derived by Boussinesq spawned a variety of models which we call "Boussinesq Paradigm". The next important step was made when Zabusky and Kruskal [11] discovered numerically that the solitary waves retain their shapes after multiple collisions. They introduced the coinage soliton in order to emphasize this particle-like behavior of the solitary (localized) waves. Nowadays, the solitons are amply called "quasi-particles". Thus the ground for a non-probabilistic interpretation of the wave mechanics has been set. 


\section{Flexural Localized Solutions. The "Flexons"}

The flexural localized waves that are solution of Eq. (6) are called in this work "flexons". For numerical example of a shape preserving interaction of sech-es in Boussinesq equations we refer the reader to our paper [12] and the references cited therein. Because of the overwhelming numerical problem we were unable until now to come up with stable and fast enough difference scheme in order to examine the full-fledged interaction of two solitons/quasi-particles in 2D. But we have already obtained results for the shapes of stationary propagating solitons for the GDWE Eq. (6) in 2D. In the moving frame, Eq. (6) reads

$$
c_{1}^{2} \frac{\partial^{2} w}{\partial x^{2}}+c_{2}^{2} \frac{\partial^{2} w}{\partial y^{2}}+2 c_{1} c_{2} \frac{\partial^{2} w}{\partial x \partial y}=-\kappa w+\Delta\left[-w+w^{3}-\Delta w\right]
$$

where we have already specified the case with negative membrane tension when the profile of the quasi-particle is more undulated.

Fig 1 presents the result for a soliton propagating along $y$-line (right panel). Comparing the result to the shape of a resting soliton (left) shows that the moving quasi-particle is contracted in the direction of motion. This is not a Lorentz contraction, however, because the speed of light is not present for the flexural deformations. Yet, the propensity for contraction can be viewed as an universal property of quasi-particles of any GDWE.
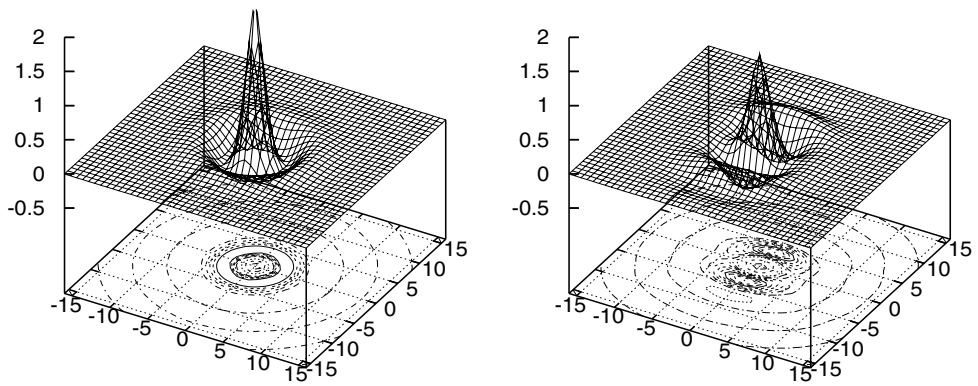

FIGURE 1. Numerical solution for the flexons of (11) with $\kappa=-0.6$. Left panel: flexon at rest. Right panel: flexon propagating with phase velocity $(0 ., 0.6)$

\section{Point Particle as a Coarse-Grain Perception of a Localized Wave.}

Consider now a single quasi-particle of shape $A(\vec{\xi}, t)$ moving in the field of an external potential $U(\vec{x})$. For the wave amplitude one gets

$$
w(\vec{x}, t)=A(\vec{\xi}, t)+U(\vec{x}),
$$


where introducing $\vec{\xi}=\vec{x}-\vec{X}(t)$ allows one to acknowledge the moving frame connected with the center of the localized structure. Then

$$
\nabla w_{t}=\nabla A_{t}-\dot{\vec{X}}(t) \cdot \nabla \nabla A ; \quad \nabla^{2} w=\nabla^{2} A+\nabla^{2} U ; \quad \nabla \Delta w=\nabla \Delta A+\nabla \Delta U .
$$

Then the wave momentum from Eq. (9) $\vec{P}$ gives the following

$$
\mathscr{P}=-\int_{D}\left(\nabla A_{t}\right) \cdot(\nabla \nabla A) \mathrm{d}^{3} \vec{x}+\frac{d}{d t} \vec{X}(t) \cdot \int_{D}(\nabla \nabla A) \cdot(\nabla \nabla A) \mathrm{d}^{3} \vec{x},
$$

which can be called pseudomomentum of the geometrical center of the solitary wave when perceived as a point particle. It is well known, however, that the shape of a soliton depends on its phase speed. If we consider the limiting case of slow acceleration/deceleration of the wave, we can neglect the first term in the right hand side and then the above equation gives that the matrix,

$$
M=\int_{D}(\nabla \nabla A) \cdot(\nabla \nabla A) \mathrm{d}^{n} \vec{x},
$$

plays the role of a mass. To establish the analogy with the classical particles we mention that the shape $A(\xi ; \vec{c})$ depends on the phase velocity of the center of the wave or pseudvelocity of the quasi-particle (see Fig. 1). When the phase speed is small, the quasiparticle will retain its almost point symmetry and the matrix $M$ will be approximately reduced to a scalar

$$
\mathscr{M}=\int_{D}(\Delta A)^{2} \mathrm{~d}^{3} \vec{x}
$$

which we will call pseudomass of a single particle in the classical limit. In a sense it is a measure of the resistance (inertia) against changing the measure of the curvature of the shape of pseudo-particle. Then the conservation law for the moment (10), yields the Newton law for the acceleration with $\mathscr{M}$ playing the role of mass, namely $\frac{d}{d t}(\mathscr{M} \dot{X})=0$.

For two quasi-particles with shapes $A_{i}$ and trajectories $\vec{X}_{i}(t)$, one has

$$
w=A_{1}\left(\vec{x}-\vec{X}_{1}(t)\right)+A_{2}\left(\vec{x}-\vec{X}_{2}(t)\right)+A_{12}\left(\vec{x}-\vec{X}_{1}(t), \vec{x}-\vec{X}_{2}(t)\right) .
$$

For well separated particles, the term $F_{12}$ can be neglected. Then

$$
\frac{\partial w}{\partial t}=-\nabla A_{1} \cdot \frac{d X_{1}}{d t}-\nabla A_{2} \cdot \frac{d X_{2}}{d t},
$$

and the term $\left(\nabla w_{t}\right)^{2}$ yields the following term in the discrete-system Hamiltonian:

$$
\sum_{i=1}^{2} \sum_{j=1}^{2} \mathscr{M}_{i j} \cdot \dot{X}_{i} \dot{X}_{j}, \quad \mathscr{M}_{i j}=\int\left(\nabla \nabla A_{i}\left(x-X_{i}\right)\right) \cdot\left(\nabla \nabla A_{j}\left(x-X_{j}\right)\right) d^{3} x,
$$

and the generalization of Newton's law involves the terms

$$
\frac{d}{d t} \mathscr{M}_{11} \dot{X}_{1}+\frac{d}{d t} \mathscr{M}_{12} \dot{X}_{2}=\ldots, \quad \frac{d}{d t} \mathscr{M}_{22} \dot{X}_{2}+\frac{d}{d t} \mathscr{M}_{21} \dot{X}_{2}=\ldots,
$$


where we can call $\mathscr{M}_{11}$ and $\mathscr{M}_{22}$ proper masses of the quasi-particles, while $\mathscr{M}_{12}=\mathscr{M}_{21}$ can be called "cross-mass" of the system of particles. For particles situated far enough from each other, one has $\left|\mathscr{M}_{12}\right| \ll \max \left|\mathscr{M}_{22}\right|,\left|\mathscr{M}_{11}\right|$ for $\left|X_{1}-X_{2}\right| \gg 1$. It is interesting to note that the last result means that we have found a kind of Machean model for the quasi-particles in which model the inertial properties of a particle are influenced by the presence of other particles in its vicinity. Naturally, when the particles are far from each other, the mutual influence is negligible.

Thus we have shown that the conservation of the wave energy and momentum one can derive conservation laws for the pseudo-energy and the pseudo-momentum, provided that the pseudomass of the quasi-particle is given by the curvature mass (13).

\section{The Effect of Shell Membrane Tension as Gravitation-like Force}

Far from the center of a particle, the shape function $w$ decays to zero and the fourth derivatives and the nonlinear term can be neglected. If we do not consider cosmological scales, we can also disregard the term proportional to $\kappa \ll 1$, the latter being proportional to the inverse of the radius of the hypershell. The linear term plays a stabilizing role preventing long-wave instability on the scale proportional to radius $R$. Respectively, on short lengths, the stability is ensured by the dispersion term (the fourth derivative). This said we can consider the intermediate scales where the membrane tension is the predominant effect.

$$
\frac{\partial^{2} w}{\partial t^{2}}= \pm \Delta w
$$

Quantitatively speaking a flexon of shape $w$ will reduce the negative tension (or will introduce positive tension) and the points will experience attractive force proportional to $\nabla w$. The stationary solution for the localized disturbance in 3D gives $w \sim|\vec{x}|^{-1}$ or $\nabla w \sim G|\vec{x}|^{-2}$ for $|\vec{x}| \rightarrow \infty$. The shape function of the quasi-particle is the gravitational potential it creates.

An inverse square law (Newton's Inverse-Square Law of Gravitation) means that the shell is three dimensional. Please note that for a planar world the asymptotic behavior of $w$ is like $\ln |\vec{x}|$ and the attractive force would follow an inverse power of $|\vec{x}|$.

In principle, measuring the attraction between two isolated elementary particles (flexons) could provide the data to identify the shell (anti)-tension ("gravitational") constant.

A final note is due here on the solutions of (11) of type of harmonic waves. They are possible for the model with positive membrane tension, but their phase speed, $c_{f}$, is defined in (4) and it differs in general from the phase speed, $c$, of the shear waves in the middle surface (speed of light in Part I). Even if the gravitational waves exist, they will be highly dispersive and one cannot say that their group speed will have much to do with the phase speed. For the model with negative membrane tension, there is no place for linear harmonic gravitational waves because the problem without the fourth derivative is elliptic in time. Hence the phase speed of propagation of small disturbances will depend strongly on the frequency. And this does not violate the postulate of limiting speed of shear waves (light) in the three laminar dimensions. The flexural waves are in 
the fourth dimension and should not to be expected to be bound by the limitation from the orthogonal three dimensions.

\section{CONCLUSIONS}

We consider a material 3D hypersurface situated in the 4D geometric space. Assuming that it is a mechanical continuum which can support momentum stresses we arrive at the notion of a hypershell. The latter is governed by a nonlinear equation of Boussinesq type whose linear part is the same as for the Euler-Bernoulli and Schrödinger's equations.

The undisturbed shape of the shell can be called "physical vacuum" and the localized flexural waves (solitons or quasi-particles) are the particles. The center of a localized deformation of space is perceived as a point particle the latter being a kind of "corsegrain" description. We show how the Hamiltonian formulation of the metadynamics of the hypershell defines the Newtonian dynamics of the centers of the phase objects (the quasi-particles). The particles are not moving through the continuum. They are propagating over its surface.

Thus, in a Universe governed by the proposed here analogy, the dualism of De Broglie is replaced by a wave-particle syncretism.

A neutral particle is a flexural localized wave (flexon) sustained by the balance between the generating effect of the membrane anti-tension and the restraining effect of the cubic nonlinearity and dispersion. It is a solution of the Generalized Dispersive Wave Equation (GDWE), or the "master equation" of the wave mechanics. A charged particle appears when a twiston (see Part I) and a flexon form a bound state.

\section{REFERENCES}

1. L. Pauling, and J. E. B. Wilson, Introduction to Quantum Mechanics With Application to Chemistry, Dover, New York, 1985.

2. C. H. Hinton, Speculations on the Fourth Dimension. Selected Writings of C. H. Hinton, Dover, New York, 1980.

3. E. Schrödinger, Annalen der Physik, 79, 743 (1926).

4. H. Neuber, ZAMM, 29, 97-xx, 142-xx (1949).

5. M. Dikmen, Theory of Thin Elastic Shells, Pitman, Boston, 1982.

6. C. I. Christov, Annuaire de L'Universite de Sofia, 89, 129-140 (1995).

7. C. I. Christov, "Discrete out of continuous: Dynamics of Phase patterns in Continua," in Continuum Models and Discrete Systems - Proceedings of CMDS8, edited by K. Markov, World Scientific, Singapore, 1996, pp. 370-394.

8. S. Timoshenko, and S. Woinowsky-Krieger, Theory of Plates and Shells, McGraw-Hill, New York, 1959.

9. J. S. Russell, "Report on the Committee on Waves," in Report of 7th Meeting (1837) of British Assoc. Adv. of Sci., Liverpool, John Murray, London, 1838, pp. 417-496.

10. J. V. Boussinesq, Journal de Mathématiques Pures et Appliquées, 17, 55-108 (1872).

11. N. J. Zabusky, and M. D. Kruskal, Phys. Rev. Lett., 15, 57-62 (1965).

12. C. I. Christov, and M. G. Velarde, J. Bifurcation \& Chaos, 4, 1095-1112 (1994). 